\title{
In situ Micro-pyrolysis of 3D Nano-printed Electron Beam Sensitive Metamaterials
}

Qing Sun ${ }^{1}$, Christian Dolle ${ }^{1}$, Chantal Kurpiers ${ }^{2}$, Ruth Schwaiger $^{3}$, Peter Gumbsch ${ }^{2,4}$ and $\underline{\text { Yolita M. }}$ Eggeler $^{1}$

${ }^{1}$ Microscopy of Nanoscale Structures \& Mechanisms (MNM), Laboratory for Electron Microscopy (LEM), Karlsruhe Institute of Technology (KIT), Karlsruhe, Germany.

${ }^{2}$ Institute for Applied Materials, Karlsruhe Institute of Technology, Karlsruhe, Germany.

${ }^{3}$ Institute of Energy and Climate Research, Forschungszentrum Jülich GmbH, Jülich, Germany.

${ }^{4}$ Fraunhofer Institute for Mechanics of Materials IWM, Freiburg, Germany.

Mechanical metamaterials are micro-and nanostructural man-made materials. They can possess unusual properties such as negative elastic moduli, negative effective mass densities and non-linear behavior due to their structural design rather than their chemical character [1]. Polymeric mechanical metamaterials can for example be fabricated by three-dimensional direct laser writing (3D-DLW). By pyrolysis (thermochemical decomposition of organic materials at high temperature in vacuum or inert atmosphere), the polymer resin can be transformed into glassy carbon. This is accompanied by shrinkage and mechanical strengthening [2]. Both, miniaturization and strengthening have great potential for a variety of applications, such as carbon micro- and nanoelectromechanical systems (C-MEMS and NEMS) [3].

However, there is a lack of systematic and in-depth understanding of the pyrolytic conversion of polymeric metamaterials into glassy carbon structures on the micro- and nanometer scale. In this work, low-voltage in situ environmental SEM (ESEM) combined with in situ TEM are employed to investigate the evolution of the structural integrity during the pyrolysis process as well as the carbonization mechanism on the micro and nanoscale. The 3D polymer structures are directly printed on a MEMS-based heating chip and the pyrolysis dynamics are tracked during in situ heating. Using this direct printing approach allows to design a realm of polymeric struts with different geometrical parameters such as different lengths, widths and thicknesses, enabling to analyze the effect of various aspect ratios during micro pyrolysis. Furthermore, our tailored printing plan for the chips is optimized such that during the in situ heating experiment only the polymer structures on one quadrant of the chip are exposed to the electron beam. Hence our streamlined experimental design maximizes the iteration efficiency with respect to multiple parameters of the in situ micro-pyrolysis investigations.

In a first step, single scaffolding struts of a large metamaterial are printed directly on the MEMS chip within the heating zone of the heating spiral. Due to an expected volume shrinkage of about $80 \%$ [2], the struts are printed on polymer springs. To have a better understanding of the temperature- dependent pyrolysis-induced shrinkage [4] we first conduct three in situ heating experiments at 400, 450, and $500{ }^{\circ} \mathrm{C}$ in an ESEM using secondary electron contrast at $10 \mathrm{kV}$. Figure 1 shows how the kinetics of the shrinkage process for struts with different lengths $(5,10,15$ and $20 \mu \mathrm{m})$ are tracked during 1-hour exposure to 400, 450 , and $500{ }^{\circ} \mathrm{C}$. The kinetics of the shrinkage process are quantified in Figure 2. Our preliminary results indicate: 1) that the strut with the shortest length (the largest surface-to-volume ratio) effectively has the highest length loss than the longer ones; 2) at the highest temperature, the shrinkage is faster and the final sizes are smaller. This may be assigned to the higher possibility of the outgassing process in polymeric structures. The complementary nanoscale study with information of structural details and changes will then be conducted using TEM to create a link between kinetics and the resulting properties. 
A thorough scale-bridging understanding of the pyrolysis process will create a better understanding of the carbonization kinetics and how to use them to improve the mechanical properties of metamaterials [5].

\section{References:}

[1] J Christensen et al, MRS Commun. 5 (2015), p. 453.

[2] J Bauer et al, Nat. Mater. 15 (2016), p. 438.

[3] S Jiang et al, J. Micromech. Microeng. 25 (2015), p. 113001.

[4] Y Liu et al, Nat. Commun. 10 (2019), p. 4340.

[5] All authors gratefully acknowledge funding by the Deutsche Forschungsgemeinschaft (DFG, German Research Foundation) under Germany's Excellence Strategy 2082/1 390761711 and sincerely thank the Carl Zeiss Foundation for financial support.

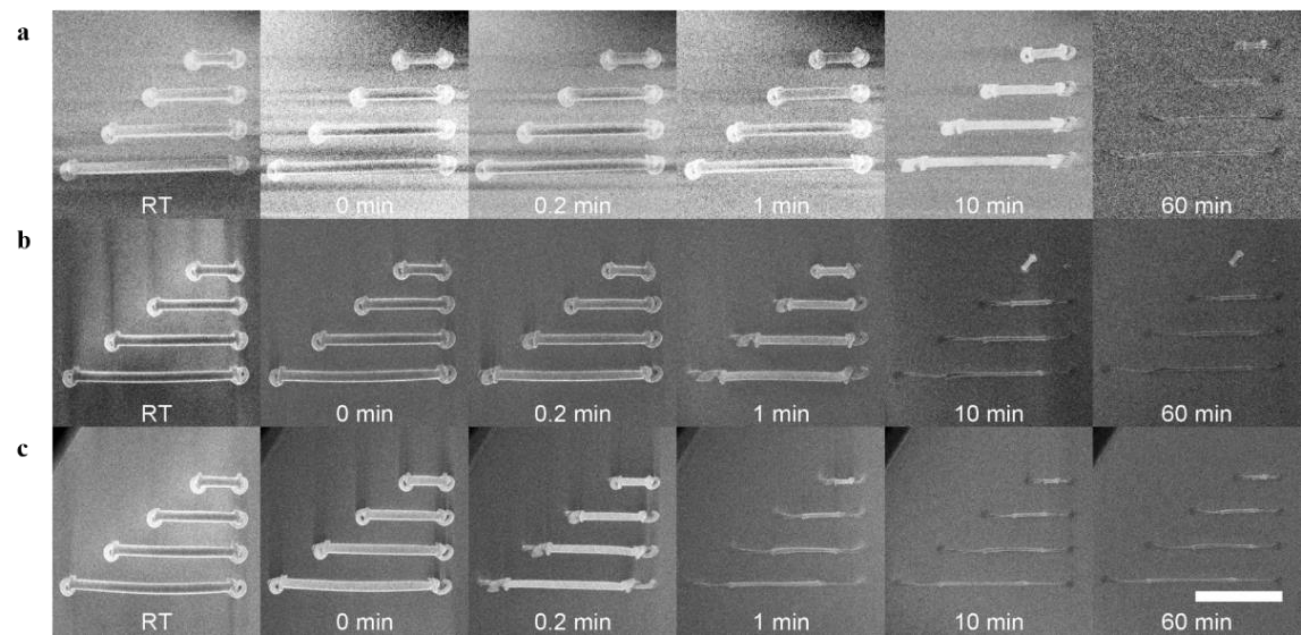

Figure 1. Series of images at different times acquired during in situ heating using secondary electron contrast in the SEM. The different strut lengths allow to investigate the effects of different lengths on the time evolution (logarithmic time) in three in situ heating experiments at (a) 400, (b) 450 and (c) $500{ }^{\circ} \mathrm{C}$. Scale bar represents $10 \mu \mathrm{m}$.
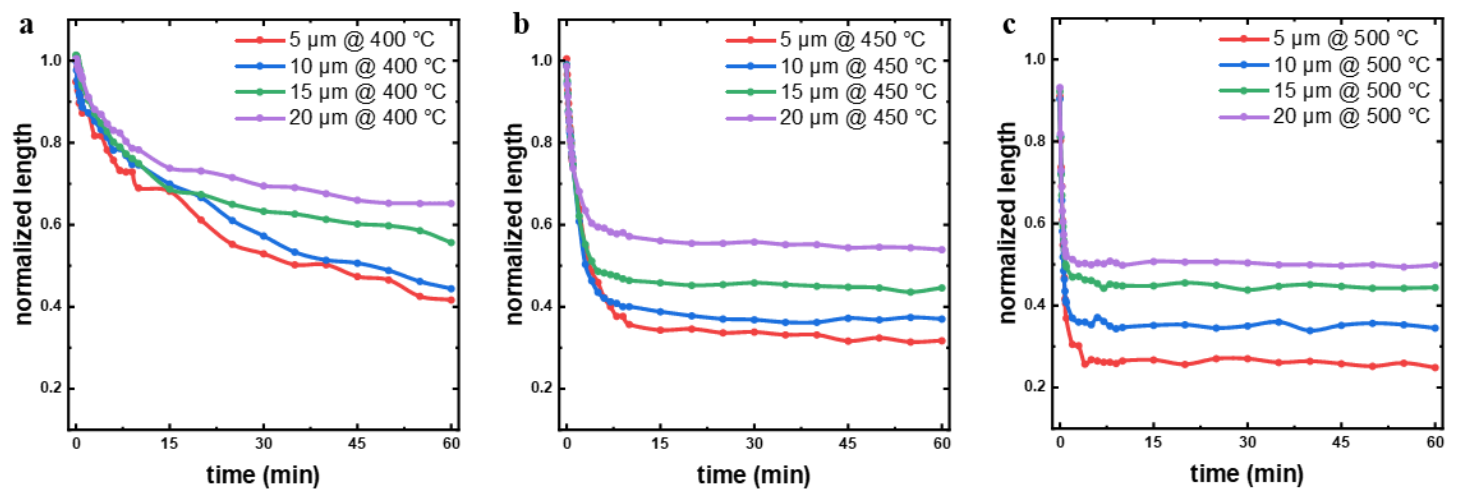

Figure 2. Quantitative analysis showing the normalized length as a function of time following the pyrolysis induced size decrease for (a) 400 , (b) 450 and (c) $500{ }^{\circ} \mathrm{C}$ and different original strut lengths (5, $10,15,20 \mu \mathrm{m})$. 